\begin{tabular}{|c} 
Ambiente \& Água - An Interdisciplinary Journal of Applied Science \\
ISSN 1980-993X - doi:10.4136/1980-993X \\
www.ambi-agua.net \\
E-mail: ambi.agua@gmail.com
\end{tabular}

\title{
Determinação e interpolação dos coeficientes das equações de chuvas intensas para cidade do Rio de Janeiro
}

\author{
ARTICLES doi:10.4136/ambi-agua.2076
}

Received: 31 Dec. 2016; Accepted: 12 Nov. 2017

\author{
Raphael Nunes de Siqueira Braga ${ }^{* *}$; Mônica de Aquino Galeano Massera da Hora²; \\ Gustavo Bastos Lyra ${ }^{3}$; Alexandre Lioi Nascentes ${ }^{4}$ \\ ${ }^{1}$ Universidade Federal Fluminense (UFF), Niterói, RJ, Brasil \\ Programa de Pós-graduação em Engenharia de Biossistemas (PGEB). E-mail: raphaelbraga03@ hotmail.com \\ ${ }^{2}$ Universidade Federal Fluminense (UFF), Niterói, RJ, Brasil \\ Departamento de Engenharia Agrícola e Meio Ambiente (TER). E-mail: dahora@vm.uff.br \\ ${ }^{3}$ Universidade Federal Rural do Rio de Janeiro (UFRRJ), Seropédica, RJ, Brasil \\ Departamento de Ciências Ambientais. E-mail: gblyra@ufrrj.br \\ ${ }^{4}$ Universidade Federal Rural do Rio de Janeiro (UFRRJ), Seropédica, RJ, Brasil \\ Departamento de Engenharia. E-mail: alexandrelioi@gmail.com \\ *Autor Correspondente
}

\section{RESUMO}

Este estudo teve como objetivo estabelecer a relação entre intensidade, duração e frequência das precipitações na cidade do Rio de Janeiro, e avaliar os métodos de interpolação dos coeficientes das equações de chuvas intensas, para determinação das equações em qualquer localidade do município. Os dados de chuvas foram obtidos por meio da rede de estações pluviométricas automáticas do Sistema Alerta Rio da Fundação Instituto de Geotécnica do município do Rio de Janeiro. Foram analisadas as precipitações intensas com base em séries históricas de observações obtidas a cada 15 minutos, no período de 1997 a 2014. Determinaramse as maiores precipitações anuais para as durações de 15, 30, 60, 120, 240, 360, 720, e 1440 minutos. Os dados foram ajustados pelo método de distribuição de Gumbel e a aderência dos dados à distribuição foi avaliada pelo teste de Kolmogorov-Smirnov a 5\% de significância. A espacialização dos coeficientes foi avaliada pelos métodos de interpolação do inverso da quinta potência da distância e Krigagem ordinária. As precipitações intensas apresentaram grande variabilidade espacial. Observou-se boa relação entre os valores de precipitação intensa calculados com o uso da equação ajustada com os valores oriundos das equações determinadas em outros trabalhos sobre chuvas intensas para a cidade do Rio de Janeiro. Os valores do coeficiente de determinação $\mathrm{R}^{2}$ e erro padrão de estimativa permitiram concluir que as equações propostas podem ser utilizadas em projetos de obras hidráulicas. O método de interpolação inverso da quinta potência da distância apresentou melhor desempenho para a espacialização dos coeficientes das equações de chuvas intensas, e menores valores de erro médio percentual em 25 das 32 estações analisadas.

Palavras-chave: função IDF, inverso da quinta potência da distância, Krigagem ordinária. 


\title{
Determination and interpolation of intense rainfall equation coefficients for the city of Rio de Janeiro
}

\begin{abstract}
This study aimed to establish the relationship between the intensity, duration and frequency of precipitation in the city of Rio de Janeiro, and to evaluate the methods of interpolation of coefficients of intense rainfall equations to determine the equations in any locality of the municipality. Rainfall data were obtained through the automatic rain gauge network of the Rio Alerta System of the Geotechnical Institute Foundation of Rio de Janeiro. Intense precipitation was analyzed based on historical series of observations obtained every 15 minutes from 1997 to 2014. The highest annual precipitations were determined for durations of 15, 30, 60, 120, 240, 360, 720, and 1440 minutes. The data were fitted by the Gumbel distribution method and adherence of the data to the distribution was evaluated by the Kolmogorov-Smirnov test at 5\% significance. The spatialization of the coefficients was evaluated by the methods of inverse fifth-distance weighting and ordinary Kriging. The intense precipitations presented great spatial variability. A good relationship was observed between the values of intense precipitation calculated with the use of the equation fitted with those derived from the equations determined in other studies about heavy rainfall for the city of Rio de Janeiro. The values of the coefficient of determination $\mathrm{R}^{2}$ and standard error of estimation showed that the proposed equations can be used in hydraulic works projects. The method of inverse fifth-distance weighting presented better performance for the spatialization of the coefficients of the intense rainfall equations and lower mean error values in 25 of the 32 analyzed stations.
\end{abstract}

Keywords: IDF function, inverse fifth distance weighting, ordinary Kriging.

\section{INTRODUÇÃO}

Na cidade do Rio de Janeiro (CRJ) observa-se a ocorrência de chuvas intensas, que ocasionam enchentes, inundações, deslizamentos e escorregamentos nas áreas urbanas. $\mathrm{O}$ conhecimento dos padrões espaço-temporal dos eventos de precipitações no município é primordial para realização de um plano adequado para o ordenamento e planejamento das áreas para redução de riscos de desastres.

A caracterização das chuvas intensas é feita por intermédio de equações empíricas denominadas equações de intensidade-duração-frequência (IDF), ou equações de chuvas intensas (Cecílio et al., 2009). O conhecimento sobre estas equações apresenta grande interesse de ordem técnica nos projetos de obras hidráulicas (Silva et al., 2002).

A CRJ apresenta grande variabilidade espacial da precipitação (Dereczynski et al., 2009), e alguns dos trabalhos sobre equações de chuvas intensas para a CRJ definem uma única equação IDF para todo território do município. Os dados usados nos ajustes dessas equações são em sua maior parte baseados em séries antigas, com utilização de dados mistos de pluviômetro e pluviógrafo, em escala de tempo diária e, ou subdiária.

Pfafstetter (1957) ajustou para 98 estações pluviográficas e pluviométricas, equações de chuvas intensas para durações variando de 5 a 2880 minutos, e para a CRJ foram disponibilizadas oito equações de chuvas intensas. Acredita-se que os dados utilizados no estudo cobrem o período de 1933 a 1955 (Davis e Naghettini, 2000).

DER (Rio de Janeiro, 1989) realizou um estudo sobre chuvas no Estado do Rio de Janeiro, que tratou sobre a coleta e análise de consistência de dados de precipitação de postos meteorológicos operados por diversas organizações oficiais. Foram utilizados dados de pluviômetros e pluviógrafos e para a CRJ foram ajustadas cinco equações IDF com uso de séries parciais de precipitação que variaram de 1944 a 1980. 
Davis e Naghettini (2000) utilizaram séries parciais de precipitação observadas em 34 estações pluviográficas distribuídas por todo o território estadual, o número de anos hidrológicos em cada estação variou de 8 a 49 anos, com número médio de 21 anos de registro, e dados de 1922 a 1999. Para a CRJ foram ajustadas equações de chuvas intensas em que a intensidade é calculada de acordo com a precipitação média anual de cada localidade.

Rio-Águas (2010) elaborou um documento com instruções técnicas para elaboração de estudos hidrológicos e dimensionamento hidráulico de sistemas de drenagem urbana para a CRJ em que são disponibilizadas 12 equações IDF determinadas por diversas referências bibliográficas.

A dificuldade na obtenção das equações IDF em localidades que não são disponibilizadas pode ser contornada com a utilização de procedimentos de interpolação espacial dos coeficientes das equações de chuvas intensas obtidas nas proximidades do local de interesse (Pruski et al., 2006). Segundo Castro et al. (2010), o método Krigagem (Krig) apresenta melhor desempenho para interpolação da precipitação em diversas regiões do País. O programa Plúvio 2.1 é uma ferramenta computacional livre desenvolvida pelo Grupo de Pesquisa em Recursos Hídricos da Universidade Federal de Viçosa, e estima as chuvas intensas em qualquer localidade do Brasil com o uso do método de interpolação inverso da quinta potência da distância (IDW5) (Pruski et al., 2006). Entre as equações de referência utilizadas pelos autores do programa, três equações são oriundas dos estudos desenvolvidos na CRJ, e foram realizados nas estações de Bangu, Santa Cruz e Alto da Boa Vista.

Em decorrência da dificuldade na obtenção dos dados subdiários, a maioria dos estudos de chuvas intensas possui séries históricas inferiores àquela recomendada pela Organização Mundial de Meteorologia (OMM), que é de 30 anos (Silva et al., 2002).

Neste aspecto, existe a necessidade de revisão periódica, pelo menos uma vez em cada década, das equações IDF como abordado por Zuffo (2004), e segundo o autor devem ser utilizadas séries históricas de observações de precipitação do período mais recente. Desde 1997, a CRJ conta com o Sistema de Alerta da Fundação Instituto de Geotécnica do município do Rio de Janeiro (Geo-Rio), que informa, em intervalos de 15 minutos, os dados de chuvas acumulados.

Com base no exposto, o presente trabalho buscou o estabelecimento de equações IDF para as estações selecionadas para a CRJ com uso de séries históricas de observações de precipitação dos últimos anos e espacialização dos coeficientes $\left(\mathrm{K}, \mathrm{m}, \mathrm{t}_{0}, \mathrm{n}\right)$ com uso dos métodos de interpolação IDW5 e Krig, para determinação das equações de chuvas intensas em qualquer localidade da CRJ.

\section{MATERIAL E MÉTODOS}

A cidade do Rio de Janeiro está localizada na região Sudeste do Brasil, no Estado do Rio de Janeiro, entre as latitudes $22^{\circ} 45^{\prime} 05^{\prime \prime} \mathrm{S}$ e $23^{\circ} 04^{\prime} 10^{\prime \prime} \mathrm{S}$ e as longitudes $43^{\circ} 06^{\prime} 30^{\prime \prime} \mathrm{W}$ a $43^{\circ} 47^{\prime} 40^{\prime}$ ' W. A cidade desenvolve-se ao redor dos maciços da Tijuca, Gericinó ao norte, e da Pedra Branca à oeste, e é delimitada, ao sul pelo Oceano Atlântico, a leste pela Baía de Guanabara e a oeste pela baía de Sepetiba. A distribuição espacial do total pluviométrico anual médio mostra que os máximos se concentram junto aos três maciços existentes na cidade: Tijuca - Serra da Carioca (2200 mm), Gericinó - Serra do Mendanha (1400 mm) e Pedra Branca - Serra Geral de Guaratiba (1200 mm). Tais valores reduzem-se em direção às planícies, sendo um mínimo de $900 \mathrm{~mm}$ observado na Zona Norte da cidade (Dereczynski et al., 2009).

Os dados de precipitação utilizados neste trabalho referem-se aos observados na rede pluviométrica do Sistema Alerta Rio da Fundação Geo-Rio, Figura 1. As informações são divulgadas e disponibilizadas em formato digital no endereço eletrônico http://alertario.rio.rj.gov.br/download/.

\section{IPABH}

Rev. Ambient. Água vol. 13 n. 1, e2076 - Taubaté 2018 


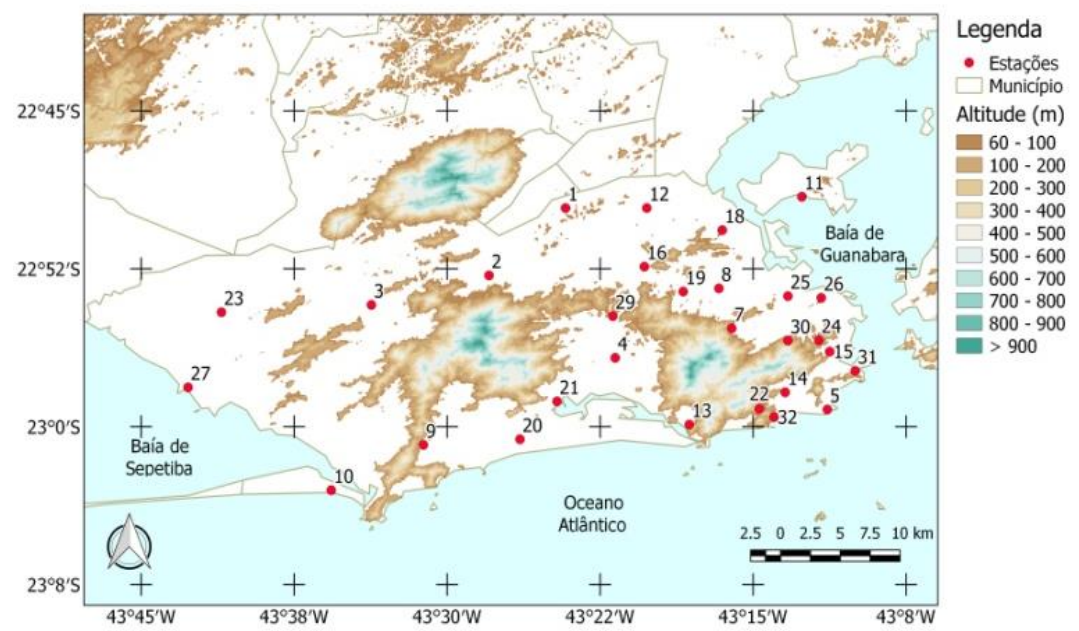

Figura 1. Distribuição espacial das estações pluviométricas automáticas do Sistema Alerta Rio selecionadas para a cidade do Rio de Janeiro. Numeração conforme Tabela 1.

Para cada estação foi selecionada uma série de dados que apresentava ausência ou pouca quantidade de falhas, sendo desconsideradas as estações com série histórica inferior a 10 anos. Do total das 37 estações da Fundação Geo-Rio, 32 atenderam a esta condição, com séries históricas de dados entre 11 e 17 anos.

Os dados obtidos foram processados e em cada ano foi identificada a maior altura pluviométrica para cada duração de chuva (série de máximos anuais). Para o estabelecimento das equações adotou-se a formulação proposta a seguir (Equação 1):

$$
I=\frac{K . T_{r}{ }^{m}}{\left(t+t_{0}\right)^{n}}
$$

em que:

I - intensidade média $\left(\mathrm{mm} \mathrm{h}^{-1}\right)$;

$\mathrm{t}$ - duração (min);

$\mathrm{T}_{\mathrm{r}}$ - tempo de retorno (anos);

$\mathrm{K}, \mathrm{m}, \mathrm{t}_{0}$ e $\mathrm{n}$ - coeficientes a determinar.

Para a CRJ, com exceção do estudo de Davis e Naghettini (2000), os demais estudos de chuvas intensas (Pfafstetter, 1957; Rio de Janeiro, 1989; Rio-Águas, 2010) utilizaram a distribuição de Gumbel para determinar a precipitação intensa provável. Portanto, as séries de intensidade máxima anual, para cada duração de chuva $(15,30,60,120,240,360,720$, e $1440 \mathrm{~min}$ ), foram ajustadas à distribuição de valores extremos de Gumbel (Equações 2, 3 e 4). A aderência dos dados foi avaliada pelo teste Kolmogorov-Smirnov, a 5\% de significância (Teodoro et al., 2014).

$$
\mathrm{X}_{\mathrm{T}}=\mathrm{X}_{\mathrm{m}}+\mathrm{K}^{*} \sigma
$$

em que:

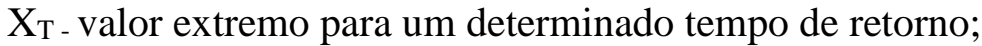

$\mathrm{X}_{\mathrm{m}}$ - valor médio da amostra;

$\sigma$ - desvio padrão da amostra; e

$\mathrm{K}$ - fator de frequência definido através da seguinte equação: 


$$
\begin{aligned}
& \mathrm{K}=\frac{\left(\mathrm{y}_{\mathrm{T}}-\mathrm{y}_{\mathrm{n}}\right)}{\mathrm{S}_{\mathrm{n}}} \\
& \mathrm{y}_{\mathrm{T}}=\left(\ln \left(\ln \left(\frac{\mathrm{T}_{\mathrm{r}}}{\mathrm{T}_{\mathrm{r}}-1}\right)\right)\right)
\end{aligned}
$$

em que:

ут - variável reduzida;

$\mathrm{y}_{\mathrm{n}}$ - média reduzida;

$\mathrm{S}_{\mathrm{n}}$ - desvio padrão reduzido;

$\mathrm{T}_{\mathrm{r}}$ - tempo de retorno (anos).

Os valores de $y_{n}$ e $S_{n}$ são fornecidos pelas Tabelas de Subramanya (2008) em função do tamanho da amostra (n). Os coeficientes $\mathrm{K}, \mathrm{m}, \mathrm{t}_{0}$ e $\mathrm{n}$ foram estimados pelo método dos mínimos quadrados com o auxílio da ferramenta Solver da planilha eletrônica Excel ${ }^{\circledR}$.

De acordo com Teodoro et al. (2014), o ajuste dos coeficientes da equação IDF pode ser avaliado por meio do coeficiente de determinação $\left(\mathrm{R}^{2}\right)$ e do erro padrão de estimativa (EPE), em $\mathrm{mm} \mathrm{h}^{-1}$, sendo este último expresso pela Equação 5:

$$
\mathrm{EPE}=\sqrt{\frac{\sum\left(\mathrm{I}_{\mathrm{c}}-\mathrm{I}_{\mathrm{e}}\right)^{2}}{\mathrm{n}}}
$$

em que:

$\mathrm{I}_{\mathrm{c}}$ - intensidade calculada pela função de distribuição de Gumbel $\left(\mathrm{mm} \mathrm{h}^{-1}\right)$;

$\mathrm{I}_{\mathrm{e}}$ - intensidade estimada pela equação ajustada $\left(\mathrm{mm} \mathrm{h}^{-1}\right)$;

n - número de observações.

A partir dos resultados alcançados, foram definidas as equações IDF para as 32 estações pluviométricas selecionadas. Estimou-se, para cada período de retorno de 2, 5, 10, 20, 50 e 100 anos, a precipitação máxima com duração de 15, 30, 60, 120, 240, 360, 720 e 1440 min.

Em seguida, as precipitações intensas, calculadas pelas equações definidas, foram comparadas com as precipitações intensas obtidas a partir das equações estabelecidas por Pfafstetter (1957), DER (1989), Davis e Naghettini (2000), Rio-Águas (2010) e Plúvio 2.1, em que foram considerados as durações e os períodos de retorno citados anteriormente, com exceção dos trabalhos de Pfafstetter (1957) e Davis e Naghettini (2000), em que não foram consideradas as durações de 360 e 720 min, pois as equações de chuvas intensas possuem expressões matemáticas distintas à do modelo da Equação 1.

Para cada estação foi calculado o erro médio percentual (EP) entre o valor estimado pela equação ajustada e o valor obtido pelas equações determinadas pelos trabalhos anteriormente citados, a fim de verificar se há sub ou superestimativa dos valores de intensidade.

Segundo Cecílio e Pruski (2003), a média dos valores do erro percentual pouco significaria na determinação de um EP, proporcionado em cada estação, portanto, optou-se pelo erro médio percentual absoluto (EMP), Equação 6, calculado com o uso do módulo do erro percentual, como proposto pelos autores e por Cecílio et al. (2009).

$$
\mathrm{EMP}=\frac{\sum_{\mathrm{i}=1}^{\mathrm{n}} \frac{\left|\mathrm{I}_{\mathrm{e}}-\mathrm{I}\right|}{\mathrm{I}_{\mathrm{e}}}}{\mathrm{n}} 100
$$

em que:

I - intensidade calculada pelas equações determinadas por outros autores.

\section{IPABH}

Rev. Ambient. Água vol. 13 n. 1, e2076 - Taubaté 2018 
Após ajustados os coeficientes das equações IDF, foi realizada a espacialização dos coeficientes $\left(\mathrm{K}, \mathrm{m}, \mathrm{t}_{0}\right.$ e $\left.\mathrm{n}\right)$ para toda a CRJ. A espacialização foi efetivada com auxílio do software ArcGIS ${ }^{\odot}$, versão 10.1, no qual foram avaliados os métodos de interpolação inverso da quinta potência da distância (IDW5), e o método de interpolação Krigagem ordinária (Krig), com modelo de transformação do tipo logarítmica de $2^{\mathrm{a}}$ ordem, e modelos de semivariograma do tipo exponencial, esférico e Gaussiano.

No método inverso da potência da distância o valor atribuído à célula interpolada é obtido pela média ponderada que utiliza o peso dos postos de controle mais próximos (Cecílio e Pruski, 2003), conforme apresentado na Equação 7.

$$
G_{i}=\frac{\sum_{i=1}^{n}\left(\frac{x_{i}}{d_{i}^{m}}\right)}{\sum_{i=1}^{n}\left(\frac{1}{d_{i}^{m}}\right)}
$$

em que:

$\mathrm{G}_{\mathrm{i}}$ - valor estimado na célula interpolada;

$\mathrm{X}_{\mathrm{i}}$ - valor do i-ésimo posto de controle;

$\mathrm{d}_{\mathrm{i}}$ - distância euclidiana entre o i-ésimo posto de controle e a célula interpolada; e

$\mathrm{m}$ - potência da distância euclidiana.

Para a aplicação do método Krigagem ordinária procedeu-se à análise geoestatística, visando quantificar o grau de dependência espacial dos dados, por meio de um semivariograma experimental, estimado pela Equação 8, de acordo com a metodologia descrita por Camargo et al. (2004):

$$
\hat{\gamma}(\mathrm{h})=\frac{1}{2 \mathrm{~N}(\mathrm{~h})} \sum_{\mathrm{i}=1}^{\mathrm{N}(\mathrm{h})}\left[\mathrm{z}\left(\mathrm{x}_{\mathrm{i}}\right)-\mathrm{z}\left(\mathrm{x}_{\mathrm{i}}+\mathrm{h}\right)\right]^{2}
$$

em que:

$\hat{\gamma}(\mathrm{h})$ - semivariograma estimado para uma distância h;

$\mathrm{N}(\mathrm{h})$ - número de pares de valores medidos;

$\mathrm{z}(\mathrm{x})$ e $\mathrm{z}(\mathrm{x}+\mathrm{h})$ - valores dos coeficientes medidos nos locais correspondentes.

$\mathrm{O}$ ajuste dos parâmetros dos semivariogramas, efeito pepita $\left(\mathrm{C}_{0}\right)$, patamar $\left(\mathrm{C}_{0}+\mathrm{C}_{1}\right)$, variância estrutural $\left(\mathrm{C}_{1}\right)$ e alcance $(\mathrm{a})$, foi realizado por meio da técnica de validação cruzada proposta por Camargo et al. (2004), sendo avaliados os modelos esférico (Equação 9), exponencial (Equação 10) e Gaussiano (Equação 11).

$$
\begin{aligned}
& \hat{\gamma}(\mathrm{h})=\mathrm{C}_{0}+\mathrm{C}_{1}\left[\frac{3}{2}\left(\frac{\mathrm{h} \mid}{\mathrm{a}}\right)-\frac{1}{2}\left(\frac{\mathrm{h} \mid}{\mathrm{a}}\right)^{3}\right]=\mathrm{C}_{0}+\mathrm{C}_{1}[\operatorname{esf}(|\mathrm{h}|)], 0<|\mathrm{h}| \leq \mathrm{a} \\
& \hat{\gamma}(\mathrm{h})=\mathrm{C}_{0}+\mathrm{C}_{1}\left[1-\exp \left(-\frac{\mathrm{h} \mid}{\mathrm{a}}\right)\right]=\mathrm{C}_{0}+\mathrm{C}_{1}[\exp (|\mathrm{h}|)] \\
& \hat{\gamma}(\mathrm{h})=\mathrm{C}_{0}+\mathrm{C}_{1}\left[1-\exp \left(-\frac{|\mathrm{h}|}{\mathrm{a}}\right)^{2}\right]=\mathrm{C}_{0}+\mathrm{C}_{1}[\operatorname{Gaussiano}(|\mathrm{h}|)]
\end{aligned}
$$


Segundo Castro et al. (2010) é importante avaliar o desempenho dos interpoladores para cada variável estudada. Neste estudo, os desempenhos dos métodos de interpolação foram avaliados pelo índice de dependência espacial (IDE), erro padrão de estimativa da validação cruzada (EPE), erro percentual (E) e o erro médio percentual absoluto (EMP) entre os valores de chuvas intensas obtidos com uso das equações IDF e os valores de precipitação estimados com o uso dos coeficientes interpolados.

O IDE é definido como a razão entre o efeito pepita e o patamar, Equação 12. Este índice determina faixas de dependência espacial entre a amostra, se o IDE $\leq 25 \%$, a amostra apresenta alta dependência espacial; se o IDE for entre $25 \%$ e $75 \%$, a amostra apresenta moderada dependência espacial; e se o IDE for $\geq 75 \%$ a amostra tem baixa dependência espacial (Cambardella et al., 1994). Os valores de EPE, também denominado como raiz do erro médio quadrático (Equações 13 e 14), deve ser o menor possível (Castro et al., 2010), assim como os valores de EMP (Cecílio et al., 2009), Equação 15.

$$
\begin{aligned}
& \operatorname{IDE}(\%)=\frac{\mathrm{Co}}{\mathrm{Co}+\mathrm{C}_{1}} \times 100 \\
& \mathrm{EPE}=\sqrt{\frac{\sum\left(\mathrm{O}_{\mathrm{i}}-\mathrm{E}_{\mathrm{e}}\right)^{2}}{\mathrm{n}}} \\
& \mathrm{E}=\left|\frac{\mathrm{I}_{\mathrm{e}}-\mathrm{I}_{\mathrm{i}}}{\mathrm{I}_{\mathrm{e}}}\right| 100 \\
& \mathrm{EMP}=\frac{\sum_{\mathrm{i}=1}^{\mathrm{n}} \mid \frac{\mathrm{I}_{\mathrm{e}}-\mathrm{I}_{\mathrm{i}} \mid}{\mathrm{I}}}{\mathrm{I}} \times 100
\end{aligned}
$$

em que:

Oi - valor do coeficiente ajustado;

$\mathrm{E}_{\mathrm{i}}$ - valor do coeficiente estimado pelo método de interpolação;

$\mathrm{I}_{\mathrm{i}}$ - valor da precipitação calculada com o uso dos coeficientes interpolados.

\section{RESULTADOS E DISCUSSÃO}

Verificou-se que, em média, ocorreram $0,51 \%$ de falhas nas 32 estações observadas, sendo que os registros foram inferiores a $2 \%$ do total de dados analisados. A quantidade de falhas encontradas pode ser considerada baixa, quando comparada com aquelas observadas em pluviógrafos. Alves et al. (2013) verificaram os registros de precipitação de pluviogramas, em que $45,4 \%$ dos dados apresentaram falhas.

O mês de janeiro de 2001 apresentou em média 13 dias consecutivos com falhas para todas as estações selecionadas. Considerando que este mês pertence à estação chuvosa da CRJ, optouse por retirar o ano de 2001 da série histórica.

A média das maiores observações de chuvas intensas variaram de $35 \mathrm{~mm}$ (15 min) até $220 \mathrm{~mm}(24 \mathrm{~h})$, e os valores médios acumulados em 15, 30 e $60 \mathrm{~min}$ foram de 35, 52 e $77 \mathrm{~mm}$, similares aos valores apresentados por Dereczynski et al. (2009), para o período 1997 a 2006, e iguais a 35, 51 e $75 \mathrm{~mm}$. Esses valores indicam que não ocorreram mudanças significativas no comportamento das chuvas intensas para o período analisado.

Todos os dados, ao serem submetidos ao teste de Kolmogorov-Smirnov a um nível de significância de 5\%, comprovaram a aderência dos dados ao modelo de Gumbel. Dessa forma, 
pode-se confirmar que a distribuição de Gumbel apresentou resultados eficazes no ajuste de eventos meteorológicos extremos, tal como observado por Teodoro et al. (2014).

$\mathrm{Da}$ análise dos resultados da Tabela 1, pode-se inferir que as equações IDF definidas apresentaram ajuste elevado, visto que os valores alcançados de $\mathrm{R}^{2}$ variaram entre 0,990 (estação Campo Grande) e 0,998 (estação Mendanha). Valores de R² superiores a 0,990 também foram obtidos para o ajuste das equações de chuvas intensas definidas por Silva et al. (2002).

Tabela 1. Estações pluviométricas operadas pelo Sistema Alerta Rio, coordenadas geográficas (latitude e longitude), período histórico de observações de cada estação, coeficientes das equações $\operatorname{IDF}\left(K, \mathrm{~m}, \mathrm{t}_{0}, \mathrm{n}\right)$, valores do coeficiente de determinação $\left(\mathrm{R}^{2}\right)$, e erro padrão de estimativa $\left(\mathrm{EPE}, \mathrm{mm} \mathrm{h}^{-1}\right)$ entre os dados obtidos pela função de distribuição de Gumbel e dados obtidos pelas equações ajustadas.

\begin{tabular}{|c|c|c|c|c|c|c|c|c|c|c|}
\hline $\begin{array}{l}\mathrm{N}^{\circ} \\
\text { ordem }\end{array}$ & Estação & Latitude & Longitude & Série histórica & $\mathrm{K}$ & $\mathrm{m}$ & $\mathrm{t}_{0}$ & $\mathrm{n}$ & $\mathrm{R}^{2}$ & EPE \\
\hline 1 & Anchieta & 22,83 & 43,40 & $\begin{array}{l}1997-2000 \\
2002-2014\end{array}$ & 2355,79 & 0,19 & 31,60 & 0,89 & 0,996 & 3,06 \\
\hline 2 & Bangu & 22,88 & 43,47 & $1997-20002002-2014$ & 8398,17 & 0,18 & 40,71 & 1,15 & 0,996 & 3,50 \\
\hline 3 & Campo Grande & 22,90 & 43,56 & $1997-20002002-2014$ & 3763,91 & 0,22 & 72,80 & 0,90 & 0,991 & 4,69 \\
\hline 4 & Cidade de Deus & 22,95 & 43,36 & $1997-20002002-2014$ & 1306,84 & 0,19 & 19,46 & 0,79 & 0,997 & 2,94 \\
\hline 5 & Copacabana & 22,99 & 43,19 & $1997-2000$ 2002-2014 & 2140,17 & 0,19 & 59,06 & 0,80 & 0,990 & 4,51 \\
\hline 6 & Gericinó & 22,52 & 43,35 & $1997-2000$ 2002-2009 & 11547,76 & 0,20 & 61,63 & 1,15 & 0,996 & 3,51 \\
\hline 7 & Grajaú & 22,92 & 43,27 & $1997-20002002-2014$ & 5274,55 & 0,20 & 52,18 & 0,98 & 0,995 & 4,21 \\
\hline 8 & Grande Méier & 22,89 & 43,28 & $1997-20002002-2014$ & 1080,84 & 0,20 & 13,79 & 0,74 & 0,996 & 3,92 \\
\hline 9 & Grota Funda & 23,01 & 43,52 & $1997-20002002-2014$ & 478,00 & 0,20 & 15,35 & 0,58 & 0,992 & 3,93 \\
\hline 10 & Guaratiba & 23,05 & 43,59 & $\begin{array}{c}1998-20002002-2003 \\
2005-2014\end{array}$ & 1107,14 & 0,22 & 14,35 & 0,80 & 0,995 & 3,93 \\
\hline 11 & Ilha do Governador & 22,82 & 43,21 & $1997-2000$ 2003-2014 & 1125,79 & 0,23 & 11,24 & 0,77 & 0,994 & 5,57 \\
\hline 12 & Irajá & 22,83 & 43,34 & $1997-2000$ 2002-2014 & 2181,48 & 0,18 & 40,07 & 0,85 & 0,995 & 3,15 \\
\hline 13 & Itanhangá & 23,00 & 43,30 & $1997-2000$ 2002-2012 & 1949,98 & 0,17 & 40,71 & 0,80 & 0,997 & 2,76 \\
\hline 14 & Jardim Botânico & 22,97 & 43,22 & $1997-20002002-2014$ & 1217,80 & 0,19 & 22,66 & 0,75 & 0,996 & 3,25 \\
\hline 15 & Laranjeiras & 22,94 & 43,19 & $2002-2014$ & 8518,56 & 0,21 & 60,99 & 1,10 & 0,994 & 4,25 \\
\hline 16 & Madureira & 22,87 & 43,34 & $1997-2000$ 2002-2014 & 2037,43 & 0,19 & 30,02 & 0,84 & 0,995 & 3,93 \\
\hline 17 & Mendanha & 22,49 & 43,31 & $1997-2000$ 2002-2009 & 531,79 & 0,17 & 7,06 & 0,63 & 0,998 & 2,29 \\
\hline 18 & Penha & 22,84 & 43,28 & $1998-2000$ 2002-2014 & 825,89 & 0,19 & 14,85 & 0,71 & 0,996 & 2,94 \\
\hline 19 & Piedade & 22,89 & 43,31 & $1997-20002002-2014$ & 2028,23 & 0,17 & 35,82 & 0,82 & 0,996 & 3,39 \\
\hline 20 & Recreio & 23,01 & 43,44 & $1997-20002002-2014$ & 6396,55 & 0,19 & 50,25 & 1,07 & 0,996 & 3,33 \\
\hline 21 & Riocentro & 22,98 & 43,41 & $1997-2000$ 2002-2014 & 1886,83 & 0,18 & 37,42 & 0,78 & 0,995 & 4,01 \\
\hline 22 & Rocinha & 22,99 & 43,25 & $1997-2000$ 2002-2014 & 10591,31 & 0,19 & 77,35 & 1,09 & 0,994 & 4,11 \\
\hline 23 & Santa Cruz & 22,91 & 43,68 & $1997-2000$ 2002-2014 & 6455,60 & 0,17 & 45,89 & 1,05 & 0,997 & 3,09 \\
\hline 24 & Santa Teresa & 22,93 & 43,20 & $1997-2000$ 2002-2014 & 2076,52 & 0,20 & 40,21 & 0,85 & 0,994 & 3,64 \\
\hline 25 & São Cristóvão & 22,90 & 43,22 & 2003-2004 2006-2014 & 1914,43 & 0,24 & 26,59 & 0,84 & 0,994 & 5,38 \\
\hline 26 & Saúde & 22,90 & 43,19 & $1997-2000$ 2002-2014 & 1222,52 & 0,18 & 25,00 & 0,75 & 0,996 & 3,11 \\
\hline 27 & Sepetiba & 22,97 & 43,71 & $1997-2000$ 2002-2014 & 1041,34 & 0,20 & 19,14 & 0,76 & 0,995 & 3,27 \\
\hline 28 & Sumaré & 22,57 & 43,14 & $1997-2000$ 2002-2009 & 1168,19 & 0,21 & 19,94 & 0,70 & 0,995 & 4,70 \\
\hline 29 & Tanque & 22,91 & 43,36 & $1997-2000$ 2002-2014 & 1896,25 & 0,22 & 19,49 & 0,86 & 0,995 & 4,85 \\
\hline 30 & Tijuca & 22,93 & 43,22 & $1997-2000$ 2002-2014 & 712,39 & 0,21 & 15,48 & 0,64 & 0,995 & 3,74 \\
\hline 31 & Urca & 22,96 & 43,17 & $1997-20002002-2014$ & 1999,11 & 0,19 & 31,01 & 0,87 & 0,997 & 2,72 \\
\hline 32 & Vidigal & 22,99 & 43,23 & $1997-20002002-2014$ & 2018,31 & 0,20 & 26,65 & 0,85 & 0,996 & 3,74 \\
\hline
\end{tabular}


O menor valor de EPE foi referente a estação Mendanha, $2,28 \mathrm{~mm} \mathrm{~h}^{-1}$, e o maior valor foi para a estação Ilha do Governador, $5,56 \mathrm{~mm} \mathrm{~h}^{-1}$. Esses dados comprovam o baixo valor de erro para estimação das chuvas intensas pelas equações IDF ajustadas.

Observou-se que os valores dos coeficientes $(\mathrm{K})$ e $\left(\mathrm{t}_{0}\right)$ variaram expressivamente de uma estação para outra, com coeficiente de variação de $99 \%$ para $(\mathrm{K})$ e para $\left(\mathrm{t}_{0}\right)$ foi de $55 \%$. Cecílio e Pruski (2003) verificaram grande variabilidade de (K) para o estado de Minas Gerais. Esses resultados indicam que o uso de uma única equação IDF para o cálculo de precipitações intensas em qualquer localidade da CRJ não é adequado, recomendando-se o ajuste dos coeficientes das equações de chuvas intensas para cada localidade. Com relação aos coeficientes (m) e (n), a variação foi de 9 e $17,5 \%$, respectivamente.

Em relação aos valores de chuvas intensas estimados pela equação do DER (Figura 2) verificou-se que os valores EMP foram entre 21 e $60 \%$, similares aos valores de EP entre -28 e $-55 \%$. Estes resultados podem ser justificados pela série histórica utilizada, entre os anos de 1944 e 1980, o que permite concluir que as chuvas neste período foram mais intensas que as chuvas de 1997 a 2014.

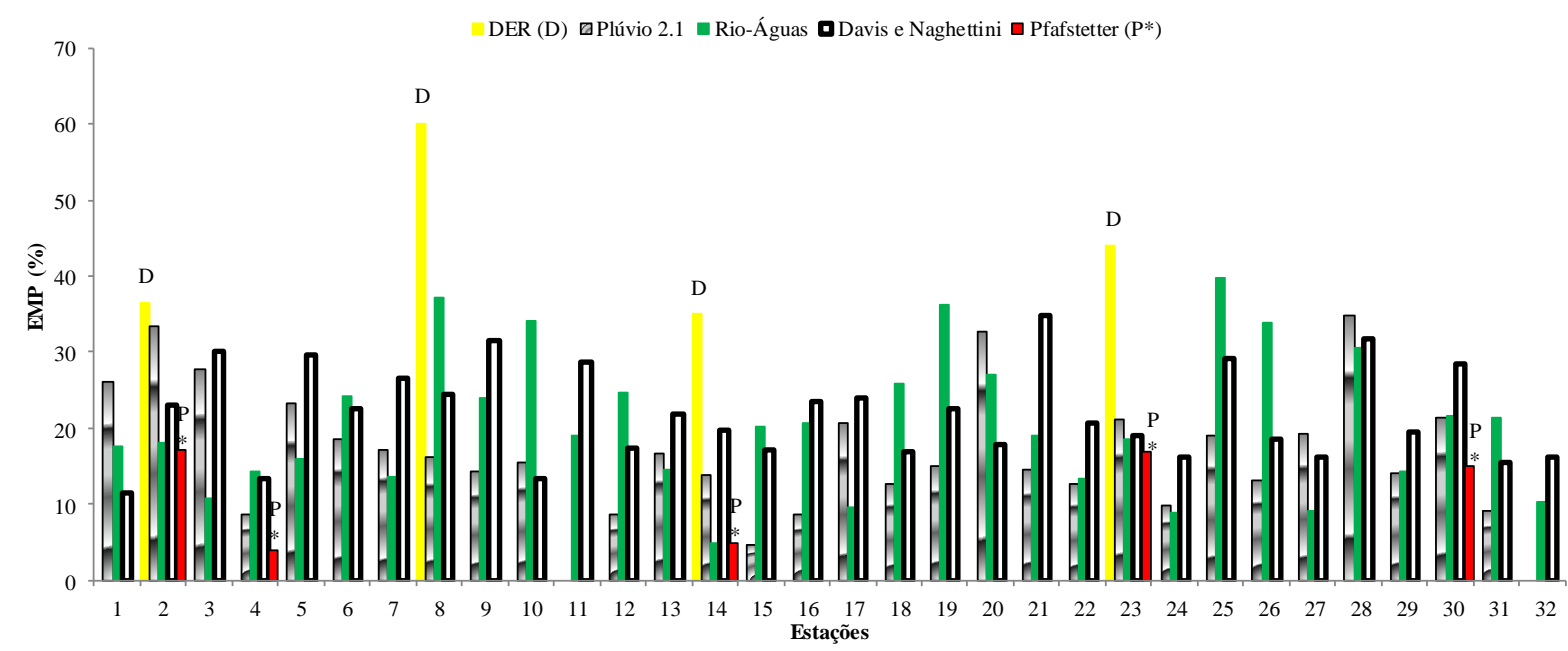

Figura 2. Erro médio percentual absoluto entre as chuvas intensas obtidas com o uso da equação ajustada e os valores de chuvas intensas calculados pelas equações determinadas em outros trabalhos para a cidade do Rio de Janeiro. Numeração conforme Tabela 1.

Verificou-se que os valores obtidos pela equação ajustada apresentaram menores erros em comparação com as equações determinadas por Pfafstetter, com valores de EMP entre 4 e 17\%, o que indicou similaridade entre as chuvas de 1933 a 1955 com o período analisado. Os valores de EP foram entre -17 e $8 \%$, e as chuvas intensas estimadas pela equação ajustada foram inferiores para as estações $2(-17 \%)$ e $4(-0,4 \%)$ e superiores para as estações $14(4 \%), 23(6 \%)$ e $30(8 \%)$.

As análises comparativas entre as equações ajustadas e as equações disponibilizadas pela Fundação Rio-Águas, em seu documento sobre instruções técnicas para elaboração de estudos hidrológicos, indicaram boa relação para 21 das 32 estações analisadas, com valores de EMP inferiores a $22 \%$. Os valores de EMP foram de 5 a $40 \%$ e os valores de EP foram entre -34 e $40 \%$. As chuvas intensas estimadas pela equação ajustada foram superiores em 17 das 32 estações observadas.

Em relação ao software Plúvio 2.1 os valores de EMP foram de 5 a 35\%, em que 24 das 30 estações analisadas apresentaram valores de EMP inferiores a 22\%. Os valores de EP foram entre -30 e $35 \%$ e as chuvas intensas estimadas pelas equações ajustadas foram superiores em 21 das 30 estações analisadas. Especificamente para a CRJ, os resultados obtidos com uso do Plúvio 2.1 foram considerados aceitáveis, porém em outras localidades do Brasil o uso do 
software não expressou os mesmos resultados, tal como pode ser verificado nos estudos de Fiorio et al. (2012) e Senna et al. (2010).

Verificou-se que os valores obtidos de EMP entre as equações ajustadas e as equações de chuvas intensas determinadas por Davis e Naghettini (2000) foram de 12 a 35\%. A título de exemplo, são apresentados os resultados das curvas IDF para a estação Jardim Botânico (Figura $3)$.
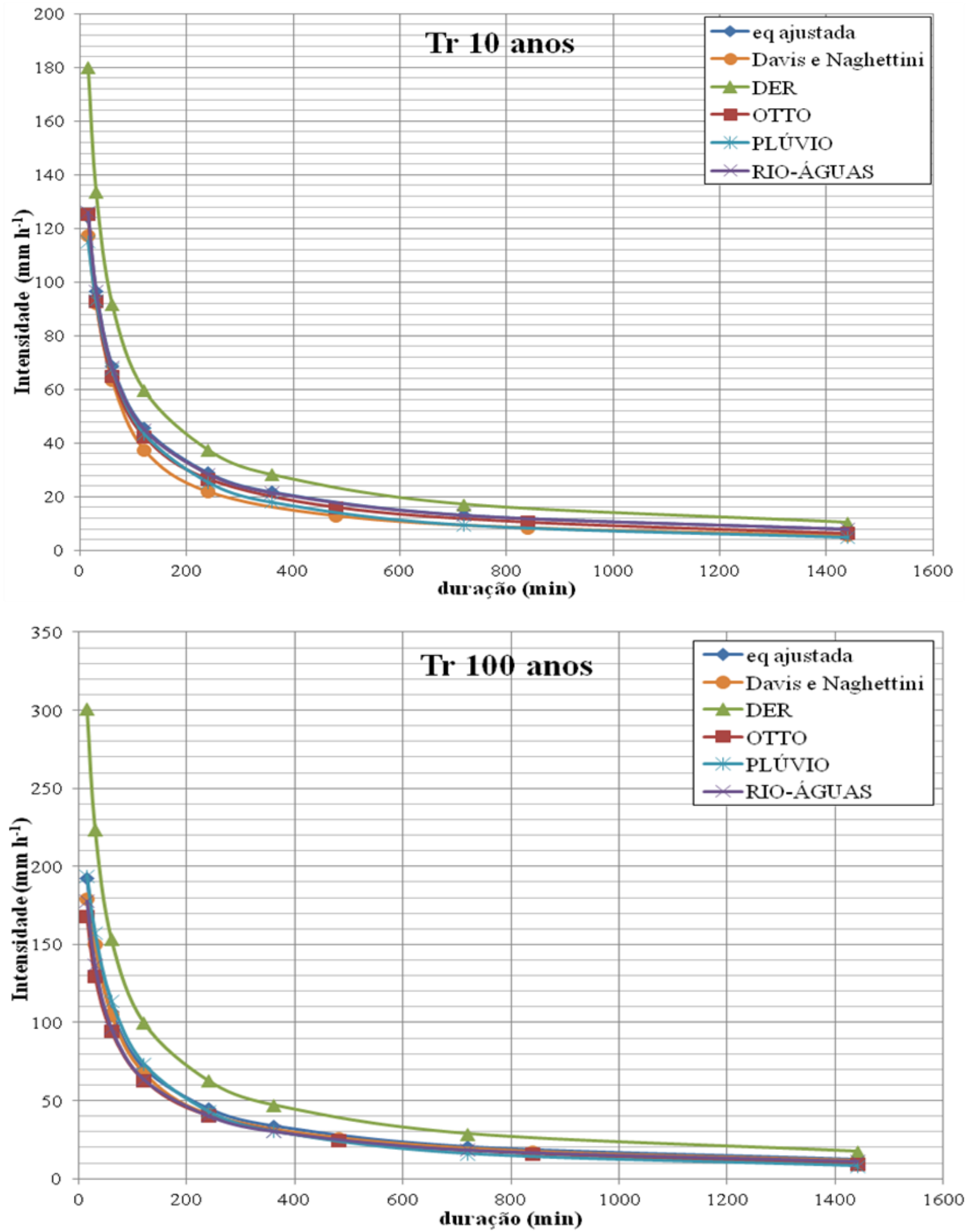

Figura 3. Comparação entre as curvas IDF geradas de acordo com as equações de chuvas intensas disponibilizadas para o Jardim Botânico. 
A estação do Jardim Botânico possui coordenadas geográficas similares entre os estudos de chuvas intensas para a CRJ. Foram verificadas as curvas IDF para o tempo de retorno de 10 e 100 anos (Zuffo, 2004). Segundo Rio-Águas (2010) para o dimensionamento de galeria de águas pluviais deve ser considerado $T_{r}$ de 10 anos, observou-se que para este $T_{r}$ a diferença entre os valores de intensidade obtidos para a duração de $15 \mathrm{~min}$ foi de $65 \mathrm{~mm} \mathrm{~h}^{-1}$ (36\%) entre o maior valor de chuva intensa, $179 \mathrm{~mm} \mathrm{~h}^{-1}$ (DER), e o menor valor de intensidade de chuva, $115 \mathrm{~mm} \mathrm{~h}^{-1}$ (Plúvio 2.1). Para a duração de $24 \mathrm{~h}$ (1440 min) a diferença entre os valores de intensidade de chuva foi de $5,5 \mathrm{~mm} \mathrm{~h}^{-1}(53 \%)$ entre o maior valor de chuva intensa, $10,5 \mathrm{~mm} \mathrm{~h}^{-1}$ (DER), e o menor valor de intensidade de chuva, $5 \mathrm{~mm} \mathrm{~h}^{-1}$ (Plúvio 2.1).

Observou-se na Figura 3 que os maiores valores de chuvas intensas foram estimados quando utilizada a equação proposta pelo DER para todos os $T_{r}$ e todas as durações analisadas. Verificou-se que para o $\mathrm{T}_{\mathrm{r}}$ de 100 anos, a diferença entre os valores de intensidade obtidos para a duração de $15 \mathrm{~min}$ foi de $131 \mathrm{~mm} \mathrm{~h}^{-1}$ (44\%) entre o maior valor de chuva intensa, $300 \mathrm{~mm} \mathrm{~h}^{-1}$ (DER), e o menor valor de intensidade de chuva, $169 \mathrm{~mm} \mathrm{~h}^{-1}$ (Pfafstetter). Para a duração de $24 \mathrm{~h}$ a diferença entre os valores de intensidade de chuva foi de $6,6 \mathrm{~mm} \mathrm{~h}^{-1}(44 \%)$ entre o maior valor de chuva intensa, $15 \mathrm{~mm} \mathrm{~h}^{-1}$ (DER), e o menor valor de intensidade de chuva, 8,4 $\mathrm{mm} \mathrm{h}^{-1}$ (Plúvio 2.1).

$\mathrm{Na}$ Tabela 2 são apresentados os valores dos índices estatísticos, IDE e EPE. Observou-se que os modelos de semivariogramas analisados apresentaram baixa dependência espacial para os coeficientes $(\mathrm{K})$ e $\left(\mathrm{t}_{0}\right)$, e moderada dependência espacial para os coeficientes $(\mathrm{n})$ e $(\mathrm{m})$, com exceção do modelo de semivariograma exponencial que apresentou alta dependência espacial para o coeficiente $(\mathrm{m})$.

Tabela 2. Métodos de interpolação e os índices estatísticos, erro padrão de estimativa da validação cruzada (EPE) e índice de dependência espacial (IDE, \%).

\begin{tabular}{lcccccccc}
\hline \multirow{2}{*}{ Métodos de interpolação } & \multicolumn{4}{c}{ EPE } & \multicolumn{4}{c}{ IDE (\%) } \\
\cline { 2 - 10 } & $\mathrm{K}$ & $\mathrm{m}$ & $\mathrm{t}_{0}$ & $\mathrm{n}$ & $\mathrm{K}$ & $\mathrm{m}$ & $\mathrm{t}_{0}$ & $\mathrm{n}$ \\
\hline Krig esférico & 3705,02 & 0,0198 & 21,99 & 0,1785 & 80 & 51 & 82 & 74 \\
Krig exponencial & 3690,87 & 0,0196 & 21,94 & 0,1782 & 85 & 5 & 84 & 74 \\
Krig Gaussiano & 3693,21 & 0,0197 & 21,98 & 0,1781 & 81 & 63 & 82 & 73 \\
IDW5 & 4000,56 & 0,0021 & 22,38 & 0,2063 & -- & -- & -- & -- \\
\hline
\end{tabular}

Segundo Cecílio et al. (2009), a aplicação da Krigagem necessita de uma distribuição mais ou menos uniforme das estações pluviométricas (pontos amostrais), fato que não foi observado neste trabalho, o que pode indicar os altos valores de IDE e a baixa dependência espacial entre os coeficientes interpolados. Porém, mesmo com os altos valores de IDE, observou-se que os valores de EPE foram menores com o uso dos métodos de interpolação de Krigagem ordinária para os coeficientes $(K, \mathrm{~m}$, e $\mathrm{n})$, apenas o coeficiente $\left(\mathrm{t}_{0}\right)$ apresentou menores valores de EPE com o uso do método IDW5.

Observou-se similaridade na maior parte dos valores dos índices estatisticos obtidos, e verificou-se que entre os métodos testados, o modelo de semivariograma exponencial apresentou melhores resultados de EPE e IDE. Castro et al. (2010) analisaram dados de precipitação para o Estado do Espirito Santo e também verificaram melhores resultados dos índices estatísticos com o uso do método de interpolação Krigagem e modelo de semivariograma exponencial.

Segundo Mello et al. (2003), as análises individuais dos coeficientes da equação IDF não apresentam significado físico, uma vez que a aplicação da equação de chuvas intensas é uma combinação de todos os coeficientes, interessando, portanto, a chuva intensa gerada pela equação como um todo. 
Cecílio e Pruski (2003) avaliaram os valores de intensidade apenas para o $\mathrm{T}_{\mathrm{r}}$ de 10 anos e duração (t) de 60 min, e o valor do erro médio encontrado para o IDW5 foi de 19,37\%, com erro percentual máximo de $88,34 \%$. O valor do erro médio para o IDW5 encontrado no presente estudo (Tabela 3) para o mesmo período de retorno e duração analisada, foi de 19,10\% com erro percentual de até $46,81 \%$. Para a Krigagem o valor do EMP para o mesmo $\mathrm{T}_{\mathrm{r}}$ e $\mathrm{t}$ foi de $24,51 \%$ com erro percentual de até $38,25 \%$. Portanto, esses valores indicam que os erros encontrados na estimativa de chuvas intensas pelos métodos de interpolação analisados neste trabalho são aceitáveis.

Tabela 3. Erros proporcionados pelos métodos de interpolação inverso da quinta potência da distância (idw5) e Krigagem ordinária (krig).

\begin{tabular}{|c|c|c|c|c|c|c|c|c|c|}
\hline \multirow{2}{*}{$\mathrm{N}^{\mathrm{o} *}$} & \multicolumn{2}{|c|}{$\operatorname{EMP}(\%)^{* *}$} & \multicolumn{2}{|c|}{$|\mathrm{E}|(\%)^{* * *}$} & \multirow{2}{*}{$\mathrm{N}^{\mathrm{o} *}$} & \multicolumn{2}{|c|}{$\operatorname{EMP}(\%)^{* *}$} & \multicolumn{2}{|c|}{$|\mathrm{E}|(\%)^{* * *}$} \\
\hline & idw5 & krig & idw5 & krig & & idw5 & krig & idw5 & krig \\
\hline 1 & 23,46 & 35,38 & 22,14 & 34,13 & 17 & 26,55 & 24,81 & 26,19 & 12,25 \\
\hline 2 & 43,95 & 47,19 & 31,46 & 35,95 & 18 & 21,92 & 28,51 & 25,50 & 32,83 \\
\hline 3 & 16,12 & 20,89 & 22,19 & 24,31 & 19 & 11,52 & 22,44 & 10,67 & 24,35 \\
\hline 4 & 12,25 & 33,72 & 15,66 & 34,76 & 20 & 33,01 & 41,66 & 20,34 & 31,31 \\
\hline 5 & 14,54 & 16,54 & 20,36 & 23,80 & 21 & 10,06 & 28,62 & 5,02 & 13,86 \\
\hline 6 & 41,13 & 32,55 & 27,05 & 24,42 & 22 & 9,11 & 24,03 & 9,60 & 16,58 \\
\hline 7 & 15,96 & 20,51 & 5,88 & 13,36 & 23 & 33,17 & 33,31 & 22,33 & 25,28 \\
\hline 8 & 10,27 & 18,09 & 14,29 & 21,74 & 24 & 13,71 & 31,39 & 14,32 & 31,48 \\
\hline 9 & 28,13 & 25,09 & 28,72 & 23,68 & 25 & 9,88 & 16,15 & 0,80 & 14,20 \\
\hline 10 & 52,79 & 27,76 & 46,81 & 18,52 & 26 & 17,50 & 27,14 & 21,68 & 29,85 \\
\hline 11 & 11,16 & 25,36 & 7,53 & 32,10 & 27 & 16,63 & 29,88 & 21,43 & 35,98 \\
\hline 12 & 9,40 & 19,24 & 10,08 & 21,13 & 28 & 13,98 & 7,84 & 16,56 & 4,11 \\
\hline 13 & 32,70 & 26,86 & 37,74 & 30,07 & 29 & 9,12 & 22,71 & 7,54 & 20,16 \\
\hline 14 & 36,58 & 23,79 & 40,57 & 27,68 & 30 & 16,33 & 16,84 & 24,06 & 25,02 \\
\hline 15 & 12,36 & 31,18 & 8,43 & 21,02 & 31 & 24,15 & 42,04 & 28,06 & 38,25 \\
\hline 16 & 2,56 & 14,60 & 0,84 & 15,25 & 32 & 13,14 & 29,76 & 17,38 & 27,00 \\
\hline
\end{tabular}

*Numeração conforme Tabela 1. **Erro médio percentual absoluto para todas as durações e período de retorno analisado. ***Módulo do erro percentual para a duração de 60 min e período de retorno de 10 anos.

Observa-se na Tabela 3 que o método IDW5 promoveu menores erros em 25 das 32 estações analisadas. O melhor desempenho obtido pelo método de interpolação IDW5 pode ter ocorrido devido a grande variabilidade dos coeficientes da equação IDF, com coeficiente de variação igual a 99\% (K) e 55\% ( $\left.\mathrm{t}_{0}\right)$. Cecílio e Pruski (2003) verificaram que a interpolação do parâmetro $(\mathrm{K})$ foi melhor realizada utilizando-se o inverso da quinta potência da distância, em função da grande variabilidade deste coeficiente, que foi entre 500 e 11000.

O EMP das precipitações intensas com o uso do método IDW5 foi de até 52,79\%, valor observado na estação Guaratiba. Em relação ao método de Krigagem ordinária o EMP das precipitações intensas foi de até $47,19 \%$, valor calculado para a estação Bangu.

Mello et al. (2003) consideraram como elevados valores de EMP acima de $23 \%$. O método IDW5 apresentou EMP elevado em 10 estações, e o método Krigagem ordinária apresentou EMP acima de $23 \%$ em 21 estações. Para as demais estações, os erros proporcionados pelos métodos de interpolação foram baixos, o que indicou boa exatidão na estimação de chuvas intensas com o uso dos coeficientes interpolados. 


\section{CONCLUSÃO}

Pode-se inferir que os dados obtidos pelas estações pluviométricas automáticas possibilitaram o desenvolvimento de equações de chuvas intensas similares às equações obtidas por dados pluviográficos existentes na literatura.

A interpolação dos coeficientes das equações de chuvas intensas pelo inverso da quinta potência da distância é recomendada por ser o método que proporcionou os menores valores de erro médio percentual e melhores resultados para a espacialização dos coeficientes das equações de chuvas intensas.

\section{REFERÊNCIAS}

ALVES, A. V. P.; SANTOS, G. B. S.; MENEZES FILHO, F. C. M.; SANCHES, L. Análise dos métodos de estimativa para os parâmetros das distribuições de gumbel e gev em eventos de precipitações máximas na cidade de Cuiabá-MT. Revista Eletrônica de Engenharia Civil, v. 6, n. 1, p. 32-43, 2013. http://dx.doi.org/10.5216/reec.v6i1.21635

CAMBARDELlA, C. A.; MOORMAN, T. B; NOVACK, J. M; PARKIN, T. B; KARLEN, D. L; TURCO R. F. et al. Field-scale variability of soil proprieties in central Iowa soils. Soil Science Society America Journal, v. 58, n. 5, p. 1240-1248, 1994.

CAMARGO, E. C. G.; DRUCK, S. D.; CÂMARA, G. Análise Espacial de Superfícies. In: EMBRAPA (Ed.). Análise espacial de dados geográficos. Brasília, 2004. p. 1-37.

CASTRO, F. S.; PEZZOPANE, J. E. M.; CECÍlIO, R. A.; PEZZOPANE, J. R. M.; XAVIER, A. C. Avaliação do desempenho dos diferentes métodos de interpoladores para parâmetros do balanço hídrico climatológico. Revista Brasileira de Engenharia Agrícola e Ambiental, v. 14, n. 8, p. 871-880, 2010. http://dx.doi.org/10.1590/S141543662010000800012

CECÍLIO, R. A.; PRUSKI, F. F. Interpolação dos parâmetros da equação de chuvas intensas com uso do inverso de potências da distância. Revista Brasileira de Engenharia Agrícola e Ambiental, v. 7, n. 3, p. 501-504, 2003. http://dx.doi.org/10.1590/S141543662003000300016

CECÍlIO, R. A.; XAVIER, A. C.; PRUSKI, F. F.; HOLLANDA, M. P.; PEZZOPANE, J. E. M. Avaliação de interpoladores para os parâmetros das equações de chuvas intensas no Espírito Santo. Revista Ambiente e Água, v. 4, n. 3, p. 82-92, 2009. http://dx.doi.org/10.4136/ambi-agua.298

DAVIS, E. G.; NAGHETTINI, M. C. Estudos de chuvas intensas no estado do Rio de Janeiro. 2. ed. Brasília: CPRM, 2000. 142 p.

DERECZYNSKI, C. P.; OLIVEIRA, J. S.; MACHADO, C. O. Climatologia da precipitação no município do Rio de Janeiro. Revista Brasileira de Meteorologia, v. 24, n. 1, p. 24 38, 2009. http://dx.doi.org/10.1590/S0102-77862009000100003

FIORIO, P. R.; DUARTE, S. N.; RODRIGUES, G, O.; MIRANDA, J. H.; COOKE, R. A. Comparação de equações de chuvas intensas para localidades do Estado de São Paulo. Revista Engenharia Agrícola, v. 32, n. 6, p. 1080-1088, 2012. http://dx.doi.org/10.1590/S0100-69162012000600009 
MELlO, C. R.; LIMA, J. M.; SILVA, A. M.; MELlO, J. M.; OLIVEIRA, M. S. Krigagem e inverso do quadrado da distância para interpolação dos parâmetros da Equação de chuvas intensas. Revista Brasileira de Ciência do Solo, v. 27, n. 5, p. 925-933, 2003. http://dx.doi.org/10.1590/S0100-06832003000500017

MELLO, C. R.; VIOLA, M. R.; MELLO, J. M.; SILVA, A. M. Continuidade espacial de chuvas intensas no Estado de Minas Gerais. Ciência e Agrotecnologia, v. 32, n. 2, p. 532-539, 2008. http://dx.doi.org/10.1590/S1413-70542008000200029

PFAFSTETTER, O. Chuvas intensas no Brasil. 1. ed. Rio de Janeiro: DNOS, 1957. 419 p.

PRUSKI, F. F.; SILVA, D. D.; TEIXEIRA, A. F.; CECÍlIO, R. A.; SILVA, J. M. A.; GRIEBELER, N. P. Plúvio 2.1: Chuvas intensas para o Brasil. In: PRUSKI, F. F.; SILVA, D. D.; TEIXEIRA, A. F.; CECÍLIO, R. A.; SILVA, J. M. A.; GRIEBELER, N. P. Hidros: dimensionamento de sistemas hidroagrícolas. Viçosa: Editora UFV, 2006. 259 p.

RIO DE JANEIRO (Estado). Departamento de Estradas de Rodagem - DER. Estudo de chuvas do estado do Rio de Janeiro. Rio de Janeiro: DER, 1989. 61 p.

RIO-ÁGUAS - FUNDAÇÃO INSTITUTO DAS ÁGUAS DO MUNICÍPIO DO RIO DE JANEIRO. Instruções técnicas para elaboração de estudos hidrológicos e dimensionamento hidráulico de drenagem urbana. Rio de Janeiro, 2010. 60 p.

SENNA, R. S.; XAVIER, A. C.; LIMA, J. S. S.; CECÍLIO, R. A. Metodologias para estimativa dos parâmetros da equação de chuvas intensas no Estado do Espírito Santo. Engenharia na Agricultura, v. 18, n. 6, p. 496-503, 2010. http://dx.doi.org/10.13083/14143984.v18n06a05

SILVA, D. D.; GOMES FILHO, R. R.; PRUSKI, F.; PEREIRA, S. B.; NOVAES, L. F. Chuvas intensas para o Estado da Bahia. Revista Brasileira de Engenharia Agrícola e Ambiental, v. 6, n. 2, p. 362-367, 2002. http://dx.doi.org/10.1590/S141543662002000200030

SUBRAMANYA, K. Engineering Hydrology. 3. ed. New Delhi: Tata McGraw-Hil, 2008. 434 p.

TEODORO, P. E.; NEIVOCK, M. P.; MARQUES, J. R. F.; FLORES, A. M. F.; RODRIGUES, C. C. B. Influência de diferentes coeficientes de desagregação na determinação de equações idf para Aquidauana/MS. Revista Eletrônica de Engenharia Civil, v. 9, n. 2, p. 1-9, 2014. http://dx.doi.org/10.5216/reec.v9i2.28701

ZUFFO, A. C. Equações de chuvas são eternas? In: CONGRESSO LATINO-AMERICANO DE HIDRÁULICA, 21, 18-22 out. 2004, São Pedro. Anais... São Pedro: IARH, 2004. p. $1-9$. 